\title{
XVI. On the equilibrium of arches
}

\section{Mr. John Southern}

To cite this article: Mr. John Southern (1801) XVI. On the equilibrium of arches, Philosophical Magazine Series 1, 11:42, 97-107, DOI: 10.1080/14786440108675963

To link to this article: http://dx.doi.org/10.1080/14786440108675963

$$
\text { 曲 Published online: } 18 \text { May } 2009 .
$$

Submit your article to this journal

III Article views: 2

Q View related articles $\asymp$ 


\section{$\left[\begin{array}{ll}9 & 97\end{array}\right]$}

XVI. On the Equilibrium of Arcbes. By Mr. Joнx So uthern, Engineer, Sobo, Birmingbam*.

SI R,

Soho, Birmingham, Nov, z, rsor.

A

T the prefent moment, when the grand project of throwing a fingle arch over the Thames (propoled by Meffrs. Telford and Douglas) is in contemplation, whatever tends to render the theory of the equilibrium of arches more eafily to be underftond, will, it is reafonable to fuppofe, be acceptable to many of your readers; and if you think the following paper of this defcription, you will, by inferting it in the Philofophical Magazine, oblige,

Mr. Tillocb. Sir,

Your obedient fervant, John SOUTHERN.

t. LET the right lines, fig. $x$. ZBCDEF be fituate in a vertical plane, and moveable about the angles $B, C, D, E$ as joints, and alro about the points $Z$ and $F$; let $Z B$ be horizontal, and let fuch weights be laid upon the joints BCDE as will keep the whole in equilibrio, the points $Z$ and $F$ not yielding: reguired what fuch weights àre, and what the forces exerted in each line.

From any point $\mathrm{B}$ in the line $\mathrm{ZB}$ draw $\mathrm{B} p$ perpendicular to that line, continue the faid line to any diftance, $a$, whence draw the line $a \mathrm{M}$ parallel to $\mathrm{B} p$; by the equilibrium of forces, the weight (reprefented by the line) aM laid upnn the joint $\mathrm{B}$ will be counterbalanced by a force $a \mathrm{~B}$ in the direction $\mathrm{ZB}$, and a force $\mathrm{MB}$ in the direction $\mathrm{MB}$, and of courfe the point $B$ remain at reft. But action and reaction being equal and contrary, the force $a \mathrm{~B}$ will not only be exerted againf $\mathrm{B}$, but alfo be returned and act againt the point $Z$; alfo the force $M B$ will be returned, and act againft the joint $\mathrm{C}$. Continue $\mathrm{BC}$ to $b$, fo that $b \mathrm{C}=\mathrm{MB}$, and from $b$ draw the line $b \mathrm{~N}$ parallel to $\mathrm{B} p$, meeting the line $\mathrm{CD}$ in $\mathrm{N}$; then will the force $b \mathrm{C}$, in the direction $\mathrm{BC}$, be juft balanced by a weight $b \mathrm{~N}$ laid upon the joint $\mathrm{C}$, and a force $\mathrm{NC}$ in the direction NC. In like manner continue the lines $\mathrm{CD}$ and $\mathrm{DE}$ to $c$ and $d$ refpectively, making the lines $c \mathrm{D}$ $=\mathrm{NC}$, and $d \mathrm{E}=\mathrm{OD}$; and from the points $c$ and $d$ drawing lines parallel to $\mathrm{B} p$ meeting the line $\mathrm{DE}$. and $\mathrm{EF}$ in $\delta$

- Communicated hy the Author.

$\frac{\text { VoL. XI. }}{\text { No. } 4 \text { I. }}$

and 
and $\mathrm{P}$; fo will the weight $c \mathrm{O}$ laid upon the joint $\mathrm{D}$, and the force $\mathrm{OD}$, balance the force $c \mathrm{D}$ in the direction $\mathrm{CD}$; and the weight $d \mathrm{P}$ laid upon the joint $\mathrm{E}$, and the force $\mathrm{PE}$ balance the force $d \mathrm{E}$ acting in the direction $\mathrm{DE}$. The force $\mathrm{PE}$ will, by its reaction, be exerted upon the point $\mathrm{F}$.

Make $\mathrm{BA}=a \mathrm{~B}$, and draw $\mathrm{A} m$ parallel to $\mathrm{BC} ; \mathrm{B} m$ will therefore $=: a \mathrm{M}$, and $\mathrm{A} m=\mathrm{BM}$; and confequently the lines $\mathrm{AB}, \mathrm{B} m, m \mathrm{~A}$, will reprefent the forces and their direc tions of action which keep the joint $B$ in equilibrium. Draw $\mathrm{A} n$ parallel to $\mathrm{DC}$, then becaufe $b \mathrm{C}=\mathrm{MB}=m \mathrm{~A}$, and $b \mathbf{N}$ is parallel to $m n$ (included in $\mathrm{B} p$ ), the triangle $\mathrm{A} m n$ is equal and fimilar to the triangle $\mathrm{C} b \mathrm{~N}$, and confequently the lines $\mathrm{A} m, m n, n \mathrm{~A}$, reprefent the forces and their directions of action which keep the joint $C$ in equilibrium. So, Ao and $A p$, being refpectively drawn parallel to $D E$ and $E F$, the triangle $\AA n_{0}=\mathrm{D} c \mathrm{O}$, and $\mathrm{A} o p=\mathrm{E} d \mathrm{P}$; and confequently their fides reprefent the forces which keep the joints $\mathrm{D}$ and $\mathrm{E}$ refpectively in equilibrium.

2. It is obvious that the weight preffing on any joint is as the difference of the tangents of the angles which are formed by each of the legs of that joint with the horizontal line: for the weight on the joint $\mathrm{D}$ is as $c \mathrm{O}=n_{0}$; but $\mathrm{B} n$ is the tangent of the angle $n \mathrm{AB}$ (to the radius $\mathrm{AB}$ ) = the angle which the leg DC of the joint forms with the horizontal line $A B$; and $B o$ is the tangent of the angle $O A B$ (to the fame radius) $=$ the angle which the other leg DE of the joint forms with the horizontal line $A B$; confequently, $n a$ is the difference of thefe tangents.

3. It is equally obvious that the force exerted through any leg of any of the joints, and which may be called the compreffing force, is as the fecant of the angle which that leg forms with the horizontal line: for the force through the leg $F E$ is as $p A$, the force through $E D$ is as $0 \mathrm{~A}$, through $D C$ as $n \mathrm{~A}$, through $\mathrm{CB}$ as $m \mathrm{~A}$, and through $\mathrm{BA}$ as $\mathrm{BA}$; refpectively the fecants of the angles, with the radius $A B$, which each leg makes with the horizontal.

4. The horizontal force, or compreffing force at the crown, (which is conftant,) is $\mathrm{AB}=$ radius; becaufe the leg at the crown is horizontal, and therefore the angle is nothing there, the fecant of which is equal to radius.

5. If the weights which are difpofed upon any number of contiguous joints be united and laid upon one joint, and if the legs which form this joint have the fame declination from the horizontal line, and the fame forces exerted through them that the extreme legs of the two extreme joints of the faid contiguous number have, the equilibrium will ftill be exact.

Continue 
Continue the line $\mathrm{BC}$ to $\mathrm{G}$ and $e$, and the line $\mathrm{FE}$ to $\mathrm{G}$; make $\mathrm{G} e=\mathrm{MB}=$ the force acting towards $\mathrm{G}$ from $\mathrm{B}$; draw $\varepsilon g$ parallel to $\mathrm{B} p$. By conftruction, $\mathrm{G} e$ is parallel and equal to $\mathrm{A} m$, and $g \mathrm{G}$ is parallel to $p \mathrm{~A}$; therefore the triangles $\mathrm{G} e g$ and $A m p$ are equal and fimilar, and reprefent the forces which keep the joint $G$ at reft; bit the force or weight $e g=m p=$ $m n+n o+o p$ is equal to the fum of the weights upon the angles $\mathrm{CDE}$; and, by conftruction, the legs $F \mathrm{G}$ and $\mathrm{BG}$ of the joint $G$ have the fame declination from the horizontal that the extreme legs (FE, $\mathrm{BC}$ ) of the two extreme joints (EC) of the contiguous joints CDE have.

6. It is hence evident that the fum of the weights upon any number of fucceffive joints (whether they be numerous or few), beginning at the vertex, is as the tangent of the angle formed by the ultimate leg of the laft joint with the horizontal line.

7. Confequently, if the legs be extremely numerous and fhort, fo as to form a curve, or a line differing infenfibly from a curve, the whole weight preffing on that curve muft be as the tangent of the angle which a tangent to the curve at the loweft point makes with the horizontal line, hecaufe the faid tangent will coincide with the ultimate leg of the curve.

8. If the curve be the arch of a circle, the angle, which a tangent to the curve forms with the borizontal line, will be equal to that at the centre, contained between the vertical radius and that drawn to the point whence the tangent is drawñ, and may be meafured by the arch itfelf.

Let $a d f$, fig. 2 . be the arch of a circle from the centre $z$, and $z a$ vertical; draw $a \mathbf{F}$ horizontal, a tangent to the arch at $a$, which is conferuently at right angles to the radius $a z$. From any point in the arch, as $\varepsilon$, draw em a tangent to the chrve at $e$; the angle $\varepsilon m \mathrm{~F}$ is therefore that which the tangent to that point makes with the horizontal line; and becalte $e m$, one of its legs, is at right angles to $e z$, and $\mathrm{Fm}$, (the other leg,) by confirustion, at right angles to $a z$, the angle eza is equal to the angle $c m \mathrm{~F}$, and may be meafured on the arch from $a$ to $e$. So the anyle $d: z a$ may be proved to be equal to that which a tangent to the arch at $d$ would, if drawn, make with the horizontal. And fo of any other point.

9. Let the arch adf, in the laft figure, reprefent the intrados of a bridge; if it be required what weights fhould be laid upon any given portions of it to effect the equilibrium, draw through the given points bodef radii from the centre $z$ to the horizontal line at; fo are the portions $a \mathrm{~B}, \mathrm{BC}, \mathrm{CD}$, $\mathrm{DE}$, and $\mathrm{EF}$, proportional to the weights required to be laid

$$
\text { G } 2
$$


on the refpective portions $a b, b c, c d, d e$, and $e f$, of the arch. For $\mathrm{E} a$ is (7.) proportional to the whole weight on the arch $e a, \mathrm{D} a$ is proportional to the whole weight on the arch $d a$, and therefore $\mathrm{ED}$ is proportional to the weight on that portion of the arch ed correfponding with it, and is as the difference of the tangents of the angles formed by tangents to the arch at the points $e$ and $d$ with the horizontal. (2.)

IO. The horizontal force, or comprefling force at the crown, is as radius $(4)=.z a$; the compreffing force at any point, $c$ or $d$, is as $z \mathrm{C}$ and $z \mathrm{D}$ refpectively (3.); and, of courfe, the preffure on the abutment in direction of a tangent to the arch there, as $z \mathrm{~F}$.

II. It is eafy, from what has hitherto been demonftrated, to calculate from tables of tangents the actual weights which fhould be laid upon given portions of arches, provided the weight upon any one portion be given. Suppofe, for infance, an arch to contain from the fummit or crown to the abutment $30^{\circ}$, and that on the firt degree ro tons be laid, (including the weight of the arch, road, \&c.) then fay, $\mathrm{As}$ the tangent of one degree is to 10 tons, fo is the tangent of two degrees to the weight upon two degrees, from which fubtract the weight upon the firft, and there will remain the weight due to the fecond degree; and fo on.

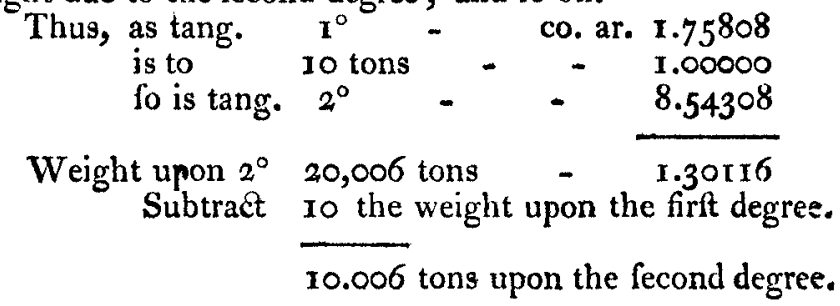

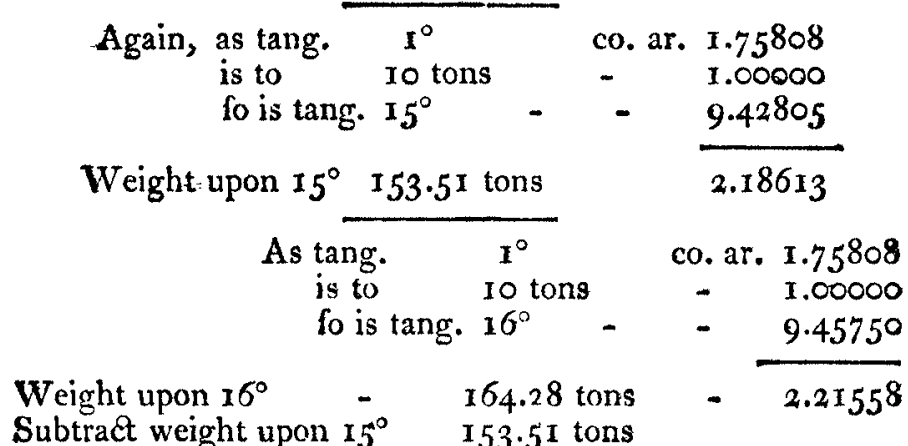

Subtract weight upon $15^{\circ} \quad 153.51$ tons

Weight upon the 16 th degree 10.77 tons 


$$
\begin{aligned}
& \begin{array}{llll}
\text { Again, as tang. } & \mathrm{I}^{\circ} & \text { co. ar. } & \mathbf{1} .75808
\end{array} \\
& \text { is to } 10 \text { tons - } 1.00000 \\
& \text { fo is tang. } 30^{\circ} \quad-\quad 9.76144
\end{aligned}
$$
Double this will be the whole weight of the bridge.

12. It was hown (3.) that the compreffing force of any leg in a fyftem of angles was as the fecant of the angle which that leg formed with the horizontal line. The leg at any point of a curve will coincide with the tangent at the fame point, and therefore the compreffing force at any point of the arch of a circle is as the fecant of the arch between that point and the vertex (8.) Thus the compreffing force at $30^{\circ}$ from the crown, is as the fecant of $30^{\circ}$; and this is the force exerted upon the abutment in direction of the tangent to the arch there. The compreffing force at $2^{\circ}$ from the crown is as the fecant of $2^{\circ}$, and at the crown (10.) as the fecant of o degrees.

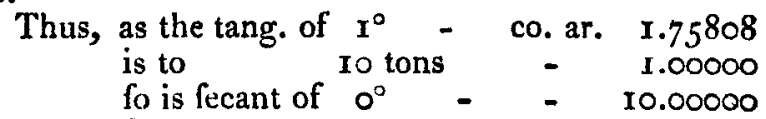

The compreffing force at the $\left.\begin{array}{l}\text { crown }=\text { the borizontal } \\ \text { force, or thruft, or puhl, } \\ \text { againft the abutment }\end{array}\right\} 572.90$ tons $-\overline{2.75808}$

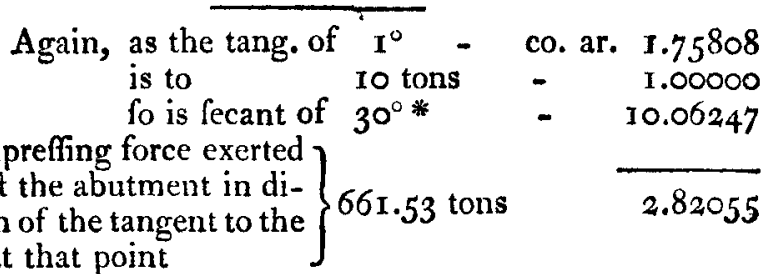

The compreffing force exerted againft the abutment in direction of the tangent to the $\} 66 \mathrm{I} \cdot 53$ tons $\quad 2.82055$ arch at that point

13. This feens to be all that is requifite to be known of the theory of the equilibrium of arches of a circle, fo far as it relates to practice : the builder will naturally defire to conftruct his bridge with the feweft materials confiftent with ftability, and will probably firft determine the dimenfions and weight of thofe at the crown, which he will fix at a minimum, and from that, by the rules juft given, the weight

* To fome of thofe who may not have a logarithmic table of fecants it may be ufeful to fay, that the logarithmic fecant of an angle may be had by fubtracting the co-line of the angle from 20.00000 ,

G 3

over 
over other parts of the arch: in moft cafes wherein the fpan of the arch is large, the fpandrils will require to be hollow, and the weights, deduced as above, will thow him how much. To carry the theory a little furrher,

14. Let $\mathrm{A} m \mathrm{~B}$, fig. 3 , be any curve equilibrated by the wall $A B C D$ built upon it, and fuftained by a horizontal force applied at $A$ the crown, and a tancential one at $B$; let $S$ be the centre of curvature at $A$ and $S A D$ vertical. It was thown 19. 10.) that the weight of incumbent matter on any portion of the arch of a circle, is to the horizontal force as the tangent of that portion of the arch is to radius. Let $A m$ be a very fmall portion of the curve, which may therefore be fuppofed not to differ fenfibly from the arch of a circle of the radius SA, nor indeed from the tangent of that arch. Therefore the weight upon $\mathrm{A} m$ will be to the horizontal force as $\mathrm{Am}$ is to radius AS: but as the weight is proportionate to the altitude $A D$ of the wall, fo is the horizontal force alfo as that altitude; and as the former may be expounded by the fmall area $\mathrm{A} n$ ( $m n$ being parallel to $\therefore \mathrm{D})=\mathrm{A} m \times \mathrm{AD}$, fo may the latter be expounded by $\mathrm{AS} \times \mathrm{AD}$ : in other words, the horizontal force, purh, or thruft, is equal to a wall whofe length is $A S$ and altitude $A D$. Make BG a tangent to the curve at $\mathrm{B}$, and draw $\mathrm{BP}$ horizontal; alfo draw sad parallel to $\mathrm{SD}$, and make $s a=\mathrm{SA}$, and $a d=\mathrm{AD}$; draw $a b$ and $d c$ horizontal, and $s b$ at right angles to BG. The angle $b s a$ is therefore equal to the angle GBP, which is the angle made by the curve at $B$ with the horizontal line; and as $a b$ is the tangent of this angle with the radius as $(=\mathrm{AS})$, the area $a b c d$ is equal to $\mathrm{ABCD}(7)=.a b \times a d$.

Draw FI parallel and very near to $\mathrm{BC}$, and $\mathrm{FH}$ a tangent to the curve at $\mathrm{F}$; alfo at right angles to $\mathrm{FH}$ draw $s f$, and from $f$ parallel to $b c$ draw $f i$. It follows, from what has been faid, that the fmall trapezium $\mathrm{BI}$ is equal to the fmall parallelogram bi.

i5. Hence the general equation for the altitude of the wall above of all curves of equilibration, which are fo poifed by the matter acting upon them verticully only, as if ftanding on vertical pillars clofe together upon the arch, viz. $a \dot{i}=w \dot{y}$, wherein

$a=$ height of the wall at the crown.

$i=$ the fluxion of the tangent of the angle GBP, formed by the tangent to any point of the curve (as $B$ ) with the horizontal line, the radius being AS.

$w=$ the altitude of the wall above the faid point (as BC), which determines the figure of the extrados.

$$
\dot{j}=
$$


$\dot{y}=$ the fluxion of the ordinate to the fame point of the curve $=$ the horizontal diftance of the lines $\mathrm{BC}$ and $\mathrm{FI}$.

16. Let the curve be the arch of a circle, and let it be required to find $w$. Let $r=$ radius, and $\dot{y}, \dot{z}$, and $\dot{t}$, the cotemporary fluxions of the fine, the arch, and the tangent, (fee fig. 4.); alfo, tet $s=$ the fecant, and $u$ be drawn parallel to $\dot{z}$.

$$
\text { then, } \begin{aligned}
& \dot{y}: \dot{z}:: r(=\mathrm{AS}): s(=\mathrm{S} b) \\
\dot{z} & : u:: r: s \\
& u: i:: r: s
\end{aligned}
$$

therefore, $\dot{y}: i:: r^{3}: s^{3}$ and $i=\frac{s^{3} y}{r^{3}}$

Subftitute this value for $i$ in the general equation above, and it will be,

$$
\begin{aligned}
& \frac{a s^{3} y}{r^{3}}=w \dot{y} ; \text { therefore } \\
& w=\frac{a s^{3}}{r^{3}} \text {. } \\
& \frac{a}{r^{3}} \text { being a confant quantity, } w \text { is proportional }
\end{aligned}
$$

to $s^{3}$, as found by others.

17. Let the curve be the parabola, fig. 5 .

A the vertex or crown.

$\mathrm{B}$ the point on which $w$ is required.

$B K$ a tangent to the curve at $B$.

$x=\mathrm{AP}$ (and by the nature of the parabola $2 x=\mathrm{PK}$ ).

$y=\mathrm{PB}$; other letters as before.

$$
\begin{aligned}
& y: 2 x:: r: t \\
& t=\frac{2 x r}{y} \\
& t=\frac{2 r y \dot{x}-2 r x \dot{y}}{y^{2}} ;
\end{aligned}
$$

and fubftituting this value for $\dot{i}$ in the general equation $a t=w \dot{y})$, we have

$$
\frac{2 a r y \dot{x}-2 a r x \dot{y}}{y^{2}}=w \dot{y} \text {, hence } w y^{2} \dot{y}=2 a r y \dot{x}-2 a r x \dot{y} .
$$

By the nature of the figure $y^{2}=p x$, where $p$ the parameter $=2 r$ ( $r$ being the radius of curvature at its vertex)

$$
\text { therefore } \begin{aligned}
y^{2}=2 r x \text { and } x & =\frac{y^{2}}{2 r} ; \\
\text { alfo } \dot{x} & =\frac{y \dot{y}}{r} .
\end{aligned}
$$


Subftituting thefe values in the equation juft above,

$$
w y^{2} \dot{y} \quad 2 a y \dot{y}-a y^{2} \dot{y}
$$

$w=a ;$ fo that the extrados and intrados are precifely fimilar and equal figures, having their vertical diftance conftantly the fame $=a$, the altitude of the wall at the crown.

18. Let the extrados be a horizontal line DC, fig. 6 , re. quired the intrados, $a$ being given, and $x, y, w$, as before.

By the figure $w=a+x$, and the general equation will be

$$
w \dot{y}=\overline{a+x} \dot{y}=a t ;
$$

therefore $\dot{y}=\frac{a \dot{i}}{a+x}$.

Alfo, $\dot{y}: \dot{x}$ (fee figure) $:: r: t$.

Therefore $\dot{y}=\frac{x \dot{x}}{t}=\frac{a \dot{t}}{a+x}$

$$
a t \dot{t}=a r \dot{x}+r x \dot{x},
$$$$
a t^{2}=2 a r x+r x^{2}
$$$$
t^{2}=\frac{x}{a} \times \overline{2 a x+x^{2}}
$$$$
t=\frac{x^{\frac{1}{2}}}{a^{\frac{1}{2}}} \sqrt{2 a x+x^{2}}
$$$$
j=\frac{x \dot{x}}{t} \text { (as above) }=\frac{x \dot{x}}{\frac{x^{\frac{1}{2}}}{a^{\frac{1}{2}}} \sqrt{2 a x+x \cdot x}}=
$$

$$
=\sqrt{a r} \frac{\dot{x}}{\sqrt{2 \dot{a} x+x x}},
$$

$$
\begin{aligned}
y= & \sqrt{a r} \times \text { hyp. log. of } \overline{a+x+\sqrt{2 a x+x x}} \\
& + \text { C. }
\end{aligned}
$$

But when $x=0, y=0$, and $C=-\sqrt{a r} \times$ hyp.log. $a$. The correct fluent is therefore

$$
y=\sqrt{a r} \times \text { hyp. } \log . \frac{a+x+\sqrt{2 a x}+x x}{a},
$$

which is the fame that Dr. Hutton makes it, page 44, new edition of his Principles of Bridges, wherein $Q$ there $=a r$, here $=$ the horizontal force.

If $a, z$, and $y$, be given in any cafe, as, for inftance, the altitude of the wall at the crown above the intrados and the 
eltitude and half fpan of the arch ; then $r$ may be found as follows, and thence the ordinates to any other point.

Let $c=$ hyp. $\log .\left(\frac{a+x+\sqrt{2 a x+x x}}{a}\right)$.

$$
\text { Then } \begin{aligned}
y & =\sqrt{a r} \times c, \\
y^{2} & =a r c^{2}, \\
r & =\frac{y^{2}}{a c^{2}} .
\end{aligned}
$$

Example. Suppofe the fpan of the arch to be roo, the altitude of it 40 , and the height of the wall above the crown 6 (fame cafe as Dr. Hutton's, page 47, Principles of Bridges),

$\dot{a}=6, x=40, y=50$, therefore

$\frac{a+x+\sqrt{2 a x+x x}}{a}=15.268$, the hyperbolic logarithm of which is $2.7257^{*}=c, c^{2}=7.4297, a c^{2}=44.578$, and $\frac{y^{2}}{a c^{2}}=\frac{2500}{44.578}=56.081=r=$ the radius of curvature of the arch at the crown.

19. The WEIGHT, of the incumbent matter on a curve loaded to the equilibrium, contained between the crown and any point $B$, fig. 3 . and which may be expounded by the area $\mathrm{AC}$ of the wall above it, has been fhown (14.) to be always $=a b \times a d=a t$. Let $\mathrm{A}=$ faid area $\mathrm{AC}$.

20. If the curve be the arch of a circle, and $T=$ the tabular tangent of the number of degrees contained in the arch, then will $t=r \mathrm{~T}$, and confequently $\mathrm{A}=a r \mathrm{~T}$.

2I. If the curve be the parabola, fee fig. 5 ;

then $y: 2 x:: r: t=\frac{2 x r}{y}$

and $\left.y^{2}=p x=2 r x--\right\}$ by the nature of the figure.

Therefore $t\left(=\frac{2 r x}{y}=\frac{y^{3}}{y}\right)=y$,

and $\mathbf{A}=y a$.

- The hyperbolic logarithm of a number may be found by multiplying the common or Briggs's logarithm of the fame number by $2.3025^{85}$, \&2. the product is the required hyperbolic logarithm. 
22. If the top or extrados be a horizontal ftraight line, fee fig. 6 ;

$$
\begin{aligned}
t & =\frac{r^{\frac{1}{2}}}{a^{\frac{1}{2}}} \sqrt{2 a x+x x}, \text { (18.) therefore } \\
t a & =\mathbf{A}=r^{\frac{1}{2}} a^{\frac{1}{2}} \sqrt{2 a x+x x}=\sqrt{\operatorname{rax} \times 2 a+x} .
\end{aligned}
$$

Example. Take the cafe of Dr. Hutton's laft mentioned, wherein $r$ was found equal to 56.081 ; therefore

$$
\begin{aligned}
& \operatorname{rax}=13459, \text { and } 2 a+x=5^{2} . \\
& \sqrt{13459 \times 5^{2}}=836.60=\mathrm{A} .
\end{aligned}
$$

23. It is well known to mechanicians that if a heavy body be fuftained by two forces, their directions muft meet either at the centre of gravity of that body, or in a vertical line which paffes through it. Let $\mathrm{A}$, fig. 7 , be the body, $g$ its centre of gravity; ea a fring by which it is fufpended, which again is fuftained by the ftrings $a b, a c$. ae is of neceffity vertical, and, if continued, muft pafs through the centre of gravity $g$ of the body. If this ftring were either longer or Shorter, the point $a$ would ftill be at reft. and the ftrings $a b$ and $a c$ under the fame tenfion. If the body were removed vertically to the fituation dotted, it would be fultained in the fame pofition by the ftrings bf and $\mathrm{cg}$, which would fuffer the fame tenfion as when they were united in the point $a$. If inftead of Atrings, props $b i, k l$, applied in the fame directions on oppofite fides of the body, were fubftituted; or if, inftead of thefe props, others parallel to them were applied, which, if continued, would meet in the vertical line, (as $m i n$ and $o p$, which meet in $g$, do,) the body would ftill be fupported at reft and in the fame pofition, and the props would fuffer the fame force of compreffion in thefe latter cafes, as the ftrings on the oppofite fides fuffered tenfion in the former.

24. Hence the CENTRE OF GRAVITY of the weights upon the joints $E, D, C$, (fig. 1.) muft be in a vertical line paffing through $\mathrm{G}$, becaufe they are fuftained in equilibrium, or at reft, by two forces in the direction $\mathrm{BC}$ and $\mathrm{FE}$, which lines, continued, meet in $\mathrm{G}$. Alfo, the centre of gravity of all the weights, fay on $B, C, D$, and $E$, mult be in a vertical line that paffes through $b$, becaufe they are fuftained by two forces in the direction FE and ZB, which, continued, meet in $b$.

25. Hence alfo the centre of gravity of the materials upon a curve in equilibrium will be in a vertical line that paffes through the point of interfection of the tangents to the extreme points of the curve. 
Let $a b c$, fig. 8 . be the curve loaded to the equilibrium; let $a d$ be a tangent to the curve at $a, c d$ a tangent to the curve at $c$, and $b_{e}$ a tangent to the curve at $b$ (the crown). The centre of gravity of the whole materials is in a vertical line which paffes through $d$; and the centre of gravity of the materials over the arch $a, b$, is in a vertical line thit pafles through the point $e$; becaufe the points $d$ and $e$ are refpectively the points of interfection of the tangents, drawn from the extreme points of the portions in queftion of the curve.

26. To find the horizontal diftance $a f$ of the centre of gravity of the materials contained between the crown and the abutment, from the latter. Drop a vertical line $e f$, from $e$, upon the ordinate $y$. Let $a f=d$, and $\dot{x}$ and $\dot{y}$ be parallel to, and cotemporary fluxions of, $x$ and $y$ refpectively. Then it is evident that $\dot{x}: \dot{y}:: x($ ef $): d(=a f)$;

$$
\text { therefore generally } d=\frac{x y}{\dot{x}} \text {. }
$$

27. If the curve be the arch of a circle, $e, b$ (fee laft figure) is evidently the tangent of half the arch, which fubtracted from half the fpan, leaves $d,=$ fine of the arch - tangent of half the arch.

28. If the curve be the parabola,

$$
y: 2 x:: \dot{y}: \dot{x}=\frac{2 x \dot{y}}{y} \text {, by the nature of that fig. }
$$

therefore $d=\frac{x \dot{y}}{\frac{2 x \dot{y}}{y}}=\frac{1}{2} y$.

29. If the curve be the equilibrial curve with a horizontal extrados.

It was thown (18) that $\dot{y}=\sqrt{a r}\left(\frac{\dot{x}}{\sqrt{2 a x+x x}}\right)=$

$$
\sqrt{ }\left(\frac{a r}{2 a x+x x}\right) \dot{x}
$$

therefore $d\left(=\frac{x \dot{y}}{\dot{x}}\right)=\sqrt{\frac{a r x x}{2 a x+x x}}=\sqrt{\frac{a r x}{2 a+x}}$.

Example. Take the cafe of Dr. Hutton's mentioned before, wherein $r$ was found $=5^{6.081}, \operatorname{rax}=134.59$, and $2 a+x=52$,

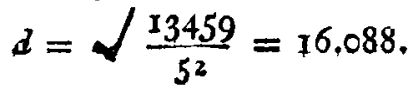

